\title{
Ethnic differences in body mass index and associated factors of adolescents from minorities in Oslo, Norway: a cross-sectional study
}

\author{
BN Kumar ${ }^{1, *}$, G Holmboe-Ottesen ${ }^{1}, \mathrm{~N}$ Lien $^{2}$ and M Wandel ${ }^{2}$ \\ ${ }^{1}$ Section of Preventive Medicine and Epidemiology, Department Group for Community Medicine and General \\ Practice, University of Oslo, PO Box 1130, N-0318 Oslo, Norway: ${ }^{2}$ Institute for Nutrition Research, University \\ of Oslo, Oslo, Norway
}

Submitted 22 July 2003: Accepted 18 May 2004

\begin{abstract}
Objectives: To examine ethnic differences in body mass index (BMI), food habits and physical activity, and determine the factors contributing to differences in BMI. Design and method: In 2000-2001, 7343 (response rate 88\%) 15- and 16-year-old students, enrolled in lower secondary schools in Oslo, participated in the crosssectional Oslo Health Study. Of these participants, 1719 were defined as ethnic minorities.

Results: Significant gender and ethnic differences in mean BMI were observed. Of the ethnic minority adolescents, 5.8\% were underweight $(<5$ th percentile of the US Centers for Disease Control and Prevention (CDC)/National Center for Health Statistics (NCHS) reference distribution) and $9.1 \%$ were overweight $(>85$ th percentile of the US CDC/NCHS reference distribution). BMI was not significantly associated with either socio-economic factors or physical activity. Food habits and physical activity differed with ethnicity but not with socio-economic factors. An ordinal regression showed that girls from East Asia (odds ratio (OR) 0.4) and boys from subSaharan Africa (OR 0.4) had lower BMI than the Western group. Among girls, higher BMI was associated with less frequent consumption of chocolates and sweets, full-fat milk and breakfast (OR 2.4, 1.7 and 1.7, respectively). Higher BMI, for both boys and girls, was associated with current and past dieting (OR 3.7 and 4.2, respectively). Conclusions: Adolescent food habits and physical activity varied by gender and ethnicity but not with socio-economic factors. BMI was associated with ethnicity, gender and food habits, but no significant relationship was observed with socioeconomic factors or physical activity. Ethnicity, in addition to gender, should be taken into consideration when studying BMI and associated factors among adolescents.
\end{abstract}

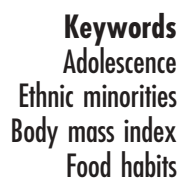

Keywords thnic minorities Food habits
Adolescence, a critical period of growth and development in the lifecycle ${ }^{1}$, establishes behavioural patterns that might predict future lifestyles. Sound eating habits formed during childhood can persist into adulthood and can prevent or delay premature onset of a number of chronic diseases ${ }^{2}$. Engeland et $a l^{3}$, among others, have shown that overweight in adolescence largely persists into adulthood.

Young people's lifestyles, including food habits and physical activity, may directly or indirectly impinge on their health both in the short term and the long term. Skipping meals, dieting and snacking are some of the issues of global concern among adolescents. Growing popularity, particularly in developed countries, with regard to television (TV) viewing, computer and video games, coupled with limited time for physical activities, has been observed ${ }^{4,5}$. There is growing evidence to suggest that lower levels of physical activity and sedentary behaviour are associated with a higher prevalence of obesity in children and adolescents ${ }^{6-8}$.

However, it is increasingly evident that the burden of disease is not shared equally by all segments of the population and evidence suggests that dietary factors, physical activity and nutrition-related diseases differ by race/ethnicity, in addition to gender and socio-economic variables $^{5,9}$

Immigration from developing countries to Norway is fairly recent, dating back 30 years. This population currently comprises first- and second-generation immigrants. The second-generation immigrants, born and brought up in Norway, find themselves at a crossroads between the majority (host) and minority (immigrant) cultures. While information about the Norwegian 
adolescent diet has become available over the past decade $\mathrm{e}^{10,11}$, to date ethnic minorities, particularly those from developing countries, have never been described separately.

In adults, ethnic differences in body mass index (BMI) and its health implications are well established ${ }^{12}$. However, in adolescence, available literature focuses on racial $^{13}$ rather than ethnic differences. Ethnic inequalities in health have scarcely been demonstrated during adolescence, whereas Bhopal ${ }^{14}$ cites several examples in adulthood and early childhood in the course of debating the importance of research into ethnicity.

The present paper describes ethnic differences in BMI, food habits and physical activity among adolescents. It also seeks to determine factors associated with BMI, including sociodemographic factors.

\section{Subjects and methods}

The Oslo Health Study, a population-based cross-sectional study conducted in 2000-2001, encompassed both adolescents and adults living in Oslo, Norway. The National Health Screening Service (now integrated into the Norwegian Institute of Public Health), in collaboration with the Municipality of Oslo and the University of Oslo, conducted the study. The study protocol was approved by the Norwegian Data Inspectorate and cleared by the Regional Committee for Medical Research Ethics. The study has been conducted in full accordance with ethical principles as per the World Medical Association Declaration of Helsinki. This paper deals with the adolescent part of the Oslo Health Study.

\section{Subjects}

According to population registers, 8435 students aged 15-16 years, enrolled in lower secondary schools in Oslo, were eligible to participate in the study. Of these, 31 special needs students were unable to fill in the questionnaires because of mental or physical handicap and 88 students had moved to another municipality before the survey started, leaving 8316 eligible for participation. Of the eligible students, 39 did not give consent and 88 withdrew prior to participation. Of the remaining, 7343 gave their written consent and met the criteria of inclusion (completion of at least one question in either of the questionnaires), attaining a final response rate of $88.3 \%$.

\section{Data collection and data-entry methods}

The Oslo School Authorities approved the study and informed all schools; thereafter, schools consented to conducting the study and informed parents in writing. Parents could withdraw their child's participation through a written refusal. The student's written consent was also obtained, prior to participation. A group of four fieldworkers (University master's students), trained to guide the youngsters, conducted the study in 70 schools over a 12-month period. They also answered queries when required. Respondents were requested to complete a main questionnaire and a supplementary questionnaire during a double school period but were not subjected to any physical or clinical examination. The data were then entered manually into computers by staff from the National Health Screening Service. A random sample of 200 questionnaires was double punched and showed high correspondence (99.9\%). This study was not conducted anonymously but the dataset available to researchers, including data obtained from registers, was encrypted to ensure confidentiality.

\section{Questionnaire}

The questionnaires developed were based on previously conducted studies in Norway, existing scientific knowledge and current needs and priorities of both the users and producers of the data. Several questions from the food and drink section were adopted from other validated questionnaires, but the final adapted version used in this study was not validated. The original questionnaire along with an official English translation can be found at www.fhi.no.

\section{Determining ethnicity}

The majority of ethnic adolescents were born in Norway and are therefore second-generation immigrants. Ethnicity was therefore determined on the basis of their parents' country of birth. Statistics Norway's ${ }^{15}$ definition of ethnic minorities, as those having both parents born in a country other than Norway, has been applied. Failure to report both parents' country of birth $(n=50)$ resulted in exclusion from analysis. In cases where the birth countries of parents differed, the mother's country of birth was selected to determine ethnic origins.

Ethnic adolescents ( $n=1719$ ) were further divided into seven broad ethnic subgroups based on common food cultures and geographic origins. It is noteworthy that not all countries from a region were equally represented in these groups. Further exclusions included those from Southern and Central America $(n=49)$, the smallest of the seven subgroups, as well as those cases with missing gender $(n=11)$, resulting from an error in data handling. Finally, 1659 participants were included in the analysis for this paper. Data on ethnic Norwegians and the comparisons between Norwegians and ethnic adolescents are forthcoming.

\section{Calculation of BMI}

BMI $\left(\mathrm{kg} \mathrm{m}^{-2}\right)$ was calculated on the basis of self-reported weight and height. Of the available methods used to classify BMI, the US Centers for Disease Control and Prevention (CDC)/National Center for Health Statistics $(\mathrm{NCHS})^{16}$ age- and sex-specific BMI reference distributions were used to identify adolescents as being 'at risk for overweight' (BMI between the 85th and 95th 
percentile), overweight (BMI $>95$ th percentile) and underweight $(\mathrm{BMI}<5$ th percentile).

\section{Sociodemographic variables}

Statistics Norway's data registers provided information on the age, gender and residential address. In addition, the Norwegian Data Inspectorate granted permission to link other data registers like parents' education to the respondents. Mother's education and geographic divisions (East-West) are therefore derived from registry data and categorised according to guidelines issued by Statistics Norway $^{17}$.

However, other sociodemographic variables such as parental occupation, employment status and social security information were obtained from the questionnaires, i.e. self-reported. The construction and rationale of social class described here is based on youth-reported parental occupation and is discussed by Hernes and Valle (forthcoming).

\section{Food habits and dieting}

The food frequency section covered nine food categories, eight drink categories, two dietary supplement categories, three meal categories, two dietary behaviour questions and six questions related to weight and height. The food habit questions have been re-coded from six to three categories: daily consumption (1-2 and 3 or more times a day), weekly consumption (1-3 and 4-6 times a week) and seldom (seldom/never and 1-3 times a month).

A fruit and vegetable index was compiled from three variables: fruit, raw vegetables/salad and fruit juice. The new variable thus obtained was re-coded into three categories ranging from low to high consumption based on the summed score. Thus the high category of the index reflects high consumption (daily to several times a day) of at least two of three original variables. Similarly, the low category reflects low consumption (seldom/never or 1-3 times a month) of at least two of the three original categories.

Breakfast skipping was defined as those not consuming breakfast daily or less than 5-6 times a week. Dieters were defined as those on a diet currently, on a diet all the time or have been on diets in the past, whereas non-dieters are those who have never dieted. However, dieting will be not be dealt with in depth in this article.

\section{Physical activity}

Of the questions related to physical activity, one related to sports and exercise out of school hours (six categories) and another related to physical inactivity, such as TV, computer games and video out of school hours (four categories), were included. The original categories were re-coded to give three categories.

\section{Data analysis}

Data were analysed using the SPSS package, version 10.0 (SPSS Inc., Chicago, IL, USA). Cross tables were analysed with the chi-square test. An ordinal logistic regression model (with BMI in categories as the dependent variable) was used to calculate odds ratios in the multivariate analysis.

\section{Results}

Mean age of the sample was 15.6 years. Age and gender distributions across ethnic groups were fairly similar. The largest ethnic group originated from the Indian subcontinent (Table 1). Ethnic distribution across categories of sociodemographic factors varied; a higher proportion from East Asia belonged to the working class (Table 2) and a higher proportion of uneducated mothers belonged to the Middle East/North Africa group.

\section{BMI}

Significant gender differences $(P<0.002)$ in mean BMI were observed (Table 3 ). Analysis of ethnic differences revealed that boys from sub-Saharan Africa and the Indian subcontinent and girls from the Indian subcontinent and East Asia had the lowest mean BMI. Boys from the

Table 1 Sociodemographic characteristics of 15- and 16-yearolds from ethnic minorities in Oslo, Norway $(n=1659)$

\begin{tabular}{lc}
\hline & Percentage \\
\hline Gender & \\
Male & 48.9 \\
Female & 51.1 \\
Ethnic group* & \\
Western countries & 4.9 \\
Eastern Europe & 8.9 \\
North Africa and Middle East & 22.8 \\
Sub-Saharan Africa & 10.5 \\
Indian subcontinent & 42.4 \\
East Asia/Pacific & 10.5 \\
Social class† & \\
I - Upper managerial & 10.3 \\
II - Technical/economic & 5.1 \\
III - Humanistic/social & 9.0 \\
IV - Non-manual workers & 9.2 \\
V - Manual workers & 31.2 \\
Not workingf & 35.1 \\
Area of residence in Oslo & \\
Outer west & 8.8 \\
Inner west & 3.3 \\
Inner east & 18.7 \\
Outer east & 69.2 \\
Mother's education§ & \\
Uneducated & 12.9 \\
Primary school & 14.8 \\
Lower secondary & 16.2 \\
Upper secondary & 37.6 \\
University and higher education & 18.5 \\
\hline
\end{tabular}

*Western countries - Western Europe, USA, Canada, Australia and New Zealand; Eastern Europe - Russia, Baltic States, Hungary, Poland, Albania, etc.; North Africa and Middle East - Algeria, Morocco, Turkey, Iran and Iraq; sub-Saharan Africa - Somalia, Ethiopia, Ghana and Uganda; Indian subcontinent - Pakistan, Sri Lanka, India, Bangladesh, Bhutan and Burma; East Asia and Pacific - Vietnam, Philippines, China, South Korea, Thailand, Laos and Indonesia.

†Based on both parents' occupation.

$\ddagger$ Includes unemployed, retired, receiving disability benefits and students. $\S$ Register data from Statistics Norway. 
Table 2 Socio-economic factors by ethnic group among 15- and 16-year-olds in Oslo, Norway

\begin{tabular}{|c|c|c|c|c|c|c|c|}
\hline & $\begin{array}{l}\text { East Asia } \\
(n=163)\end{array}$ & $\begin{array}{c}\text { Indian } \\
\text { subcontinent } \\
(n=550)\end{array}$ & $\begin{array}{c}\text { Sub-Saharan } \\
\text { Africa } \\
(n=147)\end{array}$ & $\begin{array}{c}\text { Middle } \\
\text { East/North Africa } \\
(n=346)\end{array}$ & $\begin{array}{l}\text { Eastern } \\
\text { Europe } \\
(n=139)\end{array}$ & $\begin{array}{l}\text { Western } \\
\text { countries } \\
(n=75)\end{array}$ & $P$-value $\left(\chi^{2}\right)$ \\
\hline \multicolumn{8}{|l|}{ Social class* } \\
\hline Upper \& middle class & 21.5 & 21.8 & 25.2 & 17.3 & 36.7 & 64.0 & $<0.001$ \\
\hline Working class & 54.0 & 44.4 & 38.1 & 36.4 & 42.4 & 25.3 & \\
\hline Not working $†$ & 24.5 & 33.8 & 36.7 & 46.2 & 20.9 & 10.7 & \\
\hline \multicolumn{8}{|l|}{ Mother's education $\ddagger$} \\
\hline Less than 6 years & 19.7 & 30.5 & 23.2 & 36.9 & 9.3 & 9.6 & $<0.001$ \\
\hline $6-14$ years & 57.6 & 52.9 & 68.3 & 49.0 & 63.9 & 40.4 & \\
\hline More than 15 years & 22.7 & 16.6 & 8.5 & 14.1 & 26.9 & 50.0 & \\
\hline
\end{tabular}

Note: Cross-tabulations of socio-economic factor and ethnic groups; data are expressed as percentages unless otherwise indicated.

*Based on both parents' occupation; categories modified based on categories from Table 1. Upper \& middle includes social classes I, II and III; working comprises social classes IV and V.

† Includes retired, receiving disability benefits and students.

$\ddagger$ Register data from Statistics Norway, categories based on Table 1.

Middle East and girls from Eastern Europe had the highest mean BMI.

Boys from Western countries and the Middle East and girls from Eastern Europe had greater proportions of those overweight (BMI $>85$ th percentile). On the other hand, a greater proportion of both boys and girls from sub-Saharan Africa were underweight, having BMI $<5$ th percentile (Table 3). However, relationships between socio-economic indicators and BMI were not significant.

\section{Frequency of food and drink consumption and physical activity}

Food and drink consumption differed with ethnicity (Table 4). Significant differences in fruit and vegetable consumption were observed $(P<0.001)$, with a higher proportion of the Middle East/North Africa group consuming fruits and vegetables compared with the groups from sub-Saharan Africa and the Indian subcontinent. Fewer adolescents from Western countries consumed sweets/chocolates and soft drinks/cola daily in

Table 3 Sociodemographic factors related to body mass index (BMI) of 15- and 16-year-olds from ethnic minorities in Oslo, Norway

\begin{tabular}{|c|c|c|c|c|c|c|c|}
\hline \multirow[b]{2}{*}{ Sociodemographic factor } & \multirow[b]{2}{*}{$n$} & \multicolumn{5}{|c|}{ BMI percentile* } & \multirow[b]{2}{*}{$P$-value $\left(\chi^{2}\right)$} \\
\hline & & Mean (SD) & $<5$ th & 5th-84th & 85th-94th & $>95$ th & \\
\hline \multicolumn{8}{|l|}{ Ethnicity } \\
\hline \multicolumn{8}{|l|}{ Boys } \\
\hline East Asia/Western Pacific & 75 & $20.5(2.3)$ & 2.7 & 89.3 & 8.0 & 0.0 & 0.065 \\
\hline Indian subcontinen & 289 & $20.3(2.9)$ & 7.3 & 81.0 & 8.7 & 3.1 & \\
\hline Sub-Saharan Africa & 71 & $20.1(2.9)$ & 12.7 & 81.7 & 4.2 & 1.4 & \\
\hline Middle East/North Africa & 159 & $21.4(3.1)$ & 3.8 & 81.1 & 10.7 & 4.4 & \\
\hline Eastern Europe (63) & 63 & $21.3(1.9)$ & 0.0 & 92.1 & 7.9 & 0.0 & \\
\hline Western countries & 33 & $20.8(2.4)$ & 3.0 & 81.8 & 12.1 & 3.0 & \\
\hline Total (690) & & 20.7 (2.8)† & 5.7 & 83.0 & 8.7 & 2.6 & \\
\hline \multicolumn{8}{|l|}{ Girls } \\
\hline East Asia/Western Pacific & 85 & $19.7(1.7)$ & 1.2 & 97.6 & 0.0 & 1.2 & 0.008 \\
\hline Indian subcontinent & 247 & $19.7(2.8)$ & 9.3 & 84.6 & 3.6 & 2.4 & \\
\hline Sub-Saharan Africa & 72 & $19.9(2.4)$ & 9.7 & 86.1 & 4.2 & 0.0 & \\
\hline Middle East/North Africa & 181 & $20.6(2.5)$ & 3.3 & 87.8 & 8.3 & 0.6 & \\
\hline Eastern Europe & 75 & $20.8(2.9)$ & 4.0 & 84.0 & 10.7 & 1.3 & \\
\hline Western countries & 38 & $20.5(2.7)$ & 5.3 & 86.8 & 7.9 & 0.0 & \\
\hline Total (698) & & 20.3 (2.8)† & 6.0 & 87.2 & 5.4 & 1.3 & \\
\hline \multicolumn{8}{|l|}{ Social class $\ddagger$} \\
\hline Upper \& middle class & 344 & $20.3(2.5)$ & 5.8 & 86.9 & 6.1 & 1.2 & 0.72 \\
\hline Working class & 580 & $20.5(2.8)$ & 5.7 & 85.7 & 6.7 & 1.9 & \\
\hline Not working§ & 467 & $20.6(3.0)$ & 6.0 & 83.3 & 8.2 & 2.6 & \\
\hline \multicolumn{8}{|l|}{ Mother's education } \\
\hline Less than 6 years & 271 & $20.4(2.8)$ & 6.6 & 84.7 & 6.6 & 2.2 & 0.88 \\
\hline $6-14$ years & 547 & $20.4(2.8)$ & 5.3 & 86.4 & 6.2 & 2.0 & \\
\hline More than 15 years & 193 & $20.5(2.6)$ & 4.2 & 88.0 & 6.8 & 1.0 & \\
\hline
\end{tabular}

Note: Cross-tabulations of BMI categories and sociodemographic variables; SD - standard deviation.

${ }^{*}$ Percentiles in a US reference population.

† Comparing mean BMI within ethnic groups by analysis of variance: for boys, $P=0.011$; for girls, $P=0.002$.

$\ddagger$ Based on both parents' occupation; original six categories of social class regrouped so that class 1 (upper \& middle) now comprises I, II and III; class 2 (working) now comprises IV and V; last category remains unchanged.

$\S$ Includes retired, receiving disability benefits and students. 
Table 4 Food habits (frequencies) and physical activity of 15- and 16-year-olds by ethnic group in Oslo, Norway

\begin{tabular}{|c|c|c|c|c|c|c|c|c|}
\hline Food habits and physical activity & $\begin{array}{c}\text { Total } \\
(n=1659)\end{array}$ & $\begin{array}{l}\text { East Asia } \\
(n=174)\end{array}$ & $\begin{array}{c}\text { Indian } \\
\text { subcontinent } \\
(n=703)\end{array}$ & $\begin{array}{c}\text { Sub-Saharan } \\
\text { Africa } \\
(n=173)\end{array}$ & $\begin{array}{c}\text { Middle } \\
\text { East/North Africa } \\
(n=379)\end{array}$ & $\begin{array}{l}\text { Eastern } \\
\text { Europe } \\
(n=148)\end{array}$ & $\begin{array}{l}\text { Western } \\
\text { countries } \\
(n=82)\end{array}$ & $\begin{array}{c}P \text {-value } \\
\left(\chi^{2}\right)\end{array}$ \\
\hline \multicolumn{9}{|l|}{ Fruit and vegetable index* } \\
\hline Low & 8.3 & 5.8 & 10.7 & 13.5 & 5.4 & 3.5 & 3.8 & \multirow[t]{3}{*}{$<0.001$} \\
\hline Moderate & 63.0 & 63.0 & 65.4 & 63.5 & 56.6 & 62.7 & 72.1 & \\
\hline High & 28.7 & 31.2 & 23.1 & 23.0 & 37.9 & 33.8 & 24.1 & \\
\hline \multicolumn{9}{|l|}{ Chocolates and sweets } \\
\hline Seldom $/ 1-3$ times a month & 5.5 & 8.1 & 4.8 & 6.6 & 4.6 & 4.8 & 8.5 & \multirow{3}{*}{$<0.001$} \\
\hline Weekly/1-6 times a week & 45.3 & 46.0 & 46.9 & 46.6 & 41.0 & 41.4 & 53.7 & \\
\hline Daily/once or several times a day & 49.2 & 45.9 & 48.3 & 46.8 & 53.8 & 53.8 & 37.8 & \\
\hline \multicolumn{9}{|l|}{ Cola and soft drinks } \\
\hline Seldom/never & 12.1 & 15.9 & 11.4 & 8.4 & 10.9 & 11.6 & 21.5 & \multirow[t]{3}{*}{0.14} \\
\hline Weekly/1-6 glasses a week & 41.4 & 48.3 & 42.1 & 38.3 & 35.9 & 42.5 & 49.4 & \\
\hline Daily/1-4 glasses a day & 46.6 & 35.8 & 46.5 & 53.3 & 53.2 & 45.9 & 29.1 & \\
\hline \multicolumn{9}{|l|}{ Full-fat milk } \\
\hline Seldom/never & 25.7 & 40.6 & 19.1 & 29.7 & 24.6 & 29.7 & 40.0 & \multirow{3}{*}{$<0.001$} \\
\hline Weekly/1-6 glasses a week & 28.2 & 32.0 & 26.2 & 24.8 & 31.0 & 30.3 & 30.0 & \\
\hline Daily/1-4 glasses a day & 46.1 & 27.4 & 54.7 & 45.5 & 44.4 & 40.0 & 30.0 & \\
\hline \multicolumn{9}{|l|}{ Breakfast } \\
\hline Seldom $/ 1-3$ times a month & 16.7 & 18.7 & 18.4 & 17.0 & 13.8 & 14.9 & 14.8 & \multirow[t]{3}{*}{0.329} \\
\hline Weekly/1-4 times a week & 30.6 & 34.5 & 30.6 & 27.5 & 32.6 & 29.7 & 22.2 & \\
\hline Regular/daily, 5-6 times a week & 52.6 & 46.8 & 51.1 & 55.6 & 53.6 & 55.4 & 63.0 & \\
\hline \multicolumn{9}{|l|}{ Physical activity† } \\
\hline Less than $1 \mathrm{~h}$ a week & 73.9 & 80.6 & 77.0 & 74.6 & 68.7 & 69.7 & 65.4 & \multirow[t]{3}{*}{0.04} \\
\hline $1-4 h$ a week & 20.4 & 17.0 & 17.4 & 20.5 & 24.0 & 24.1 & 28.2 & \\
\hline More than $5 \mathrm{~h}$ a week & 5.7 & 2.4 & 5.6 & 4.8 & 7.3 & 6.2 & 6.4 & \\
\hline \multicolumn{9}{|c|}{ Time spent on television/video/computert } \\
\hline $1-2 h$ a day & 36.9 & 36.8 & 35.9 & 41.5 & 36.3 & 42.0 & 36.9 & \multirow[t]{3}{*}{0.177} \\
\hline $3-5 h$ a day & 39.4 & 45.4 & 37.6 & 34.1 & 40.2 & 45.2 & 39.5 & \\
\hline More than $5 \mathrm{~h}$ a day & 23.7 & 17.8 & 26.5 & 24.4 & 23.9 & 18.5 & 18.5 & \\
\hline \multicolumn{9}{|l|}{ Dieting } \\
\hline Never dieted & 64.6 & 60.5 & 68.0 & 67.9 & 59.8 & 61.5 & 64.2 & \multirow[t]{2}{*}{0.077} \\
\hline Past and present dieters & 35.4 & 39.5 & 32.0 & 32.1 & 40.2 & 38.5 & 35.8 & \\
\hline
\end{tabular}

Note: Cross-tabulations of ethnic groups and food and drink categories; data are expressed as percentages unless otherwise indicated.

* Index constructed from three variables: fruit, raw vegetables and salad, fruit juice. Low indicates seldom/never or less than 3 times a month for at least two of the three variables. High indicates daily or several times a day for at least two of the three variables and moderate is weekly (2-6 times a week) intake for at least two of the three variables.

†Out of school hours.

comparison to other groups. More than half the adolescents from the Indian subcontinent drank full-fat milk daily. No significant ethnic differences were observed with regard to skipping breakfast (Table 4).

Three out of four adolescents were inactive (less than $1 \mathrm{~h}$ of physical activity a week out of school hours), with the East Asian group being the most inactive (Table 4). A higher proportion of adolescents from the Indian subcontinent, sub-Saharan Africa and the Middle East watched TV/video/used a computer for more than $5 \mathrm{~h}$ a day, compared with the other groups.

Physical activity and food and drink frequencies did not vary with socio-economic indicators (data not shown).

\section{Dieting}

More than half the girls (52.1\%) had either been dieting in the past or were currently dieting, compared with $18.2 \%$ of the boys. Girls from East Asia had the highest proportion of dieters (61.1\%) whereas the lowest proportion (46.3\%) was from the Indian subcontinent $(P<0.05)$ (data not shown). No associations between dieting and the frequency of consumption of chocolates, fruits and vegetables, cola drinking or physical activity were observed (data not shown). A higher proportion of dieters skipped breakfast $(P<0.001)$ than the non-dieters. Dieters consumed full-fat milk less often than did nondieters $(P<0.001)$

\section{BMI and associated factors}

Inverse relationships between BMI and fruit and vegetable index, frequency of intake of chocolates/sweets and fullfat milk were observed for girls (Table 5). Significant associations between breakfast skipping and BMI were observed (Table 5) for both boys and girls. Higher proportions of those seldom consuming breakfast were overweight. No significant associations between BMI and sedentary activities like watching TV/video/using a computer (Table 5) or sexual maturation (girls only determined by onset of menstruation) were found (data not shown). However, for boys, an association with the level of physical activity (Table 5) was observed. Significant associations between BMI and dieting for both boys and girls were observed, with higher proportions of dieters in the higher BMI categories (data not shown).

The relationship between BMI and associated factors (ethnicity, food habits, meal patterns and slimming 
Table 5 Association of food habits and physical activity with body mass index (BMI) among 15- and 16-year-olds from ethnic minorities in Oslo, Norway $(n=1659)$

\begin{tabular}{|c|c|c|c|c|c|c|c|}
\hline \multirow[b]{3}{*}{ Factor } & \multicolumn{6}{|c|}{ BMI percentile* by gender } & \multirow[b]{3}{*}{$P$-value $\left(\chi^{2}\right)$} \\
\hline & \multicolumn{3}{|c|}{ Male (M) } & \multicolumn{3}{|c|}{ Female $(F)$} & \\
\hline & $<24$ th & 24th-85th & $>85$ th & $<24$ th & 24th-85th & $>85$ th & \\
\hline \multicolumn{8}{|l|}{ Fruit and vegetable index } \\
\hline Low & 27.4 & 56.5 & 16.1 & 53.5 & 46.5 & 0 & \multirow{3}{*}{$M=0.28, F=0.001$} \\
\hline Moderate & 28.0 & 61.4 & 10.7 & 26.1 & 64.9 & 9.0 & \\
\hline High & 21.0 & 66.2 & 12.8 & 31.5 & 63.0 & 5.6 & \\
\hline \multicolumn{8}{|l|}{ Chocolates and sweets } \\
\hline Seldom $/ 1-3$ times a month & 15.2 & 69.7 & 15.2 & 23.4 & 59.6 & 17.0 & \multirow[t]{3}{*}{$\mathrm{M}=0.43, \mathrm{~F}=0.039$} \\
\hline Weekly/1-6 times a week & 25.2 & 61.8 & 13.0 & 27.0 & 65.2 & 7.8 & \\
\hline Daily/once or several times a day & 27.7 & 62.2 & 10.1 & 32.6 & 61.7 & 5.7 & \\
\hline \multicolumn{8}{|l|}{ Full-fat milk } \\
\hline Seldom/never & 20.5 & 65.8 & 13.7 & 24.2 & 66.5 & 9.3 & \multirow[t]{3}{*}{$M=0.15, F=<0.001$} \\
\hline Weekly/1-6 glasses a week & 22.6 & 66.1 & 11.3 & 23.4 & 70.3 & 6.2 & \\
\hline Daily/1-4 glasses a day & 30.2 & 59.1 & 10.7 & 40.9 & 52.5 & 6.6 & \\
\hline \multicolumn{8}{|l|}{ Cola and soft drinks } \\
\hline Seldom/never & 30.4 & 67.4 & 2.2 & 25.0 & 66.1 & 8.9 & \multirow[t]{3}{*}{$\mathrm{M}=0.30, \mathrm{~F}=0.09$} \\
\hline Weekly/1-6 glasses a week & 27.5 & 60.1 & 12.5 & 26.3 & 66.0 & 7.6 & \\
\hline Daily $/ 1-4$ glasses a day & 25.1 & 63.3 & 11.6 & 35.5 & 58.8 & 5.7 & \\
\hline \multicolumn{8}{|l|}{ Breakfast } \\
\hline Seldom/1-3 times a month & 20.5 & 56.6 & 22.9 & 20.0 & 65.9 & 14.1 & \multirow[t]{3}{*}{$M=0.006, F=0.003$} \\
\hline Weekly/1-4 times a week & 26.0 & 61.6 & 12.4 & 32.4 & 60.9 & 6.6 & \\
\hline Regular/daily, 5-6 times a week & 27.6 & 63.6 & 8.8 & 30.3 & 64.7 & 5.0 & \\
\hline \multicolumn{8}{|c|}{ Time spent on television/video/computer ${ }^{*}$} \\
\hline $1-2 \mathrm{~h}$ a day & 26.9 & 61.2 & 11.9 & 31.2 & 62.4 & 6.5 & \multirow{3}{*}{$M=0.92, F=0.25$} \\
\hline $3-5 \mathrm{~h}$ a day & 27.0 & 62.8 & 10.2 & 27.0 & 66.5 & 6.5 & \\
\hline More than $5 \mathrm{~h}$ a day & 24.8 & 62.4 & 12.7 & 31.9 & 57.5 & 10.6 & \\
\hline \multicolumn{8}{|l|}{ Physical activity* } \\
\hline Less than $1 \mathrm{~h}$ a week & 30.0 & 57.7 & 12.3 & 29.5 & 62.7 & 7.8 & \multirow{2}{*}{$M=0.01, F=0.21$} \\
\hline More than $1 \mathrm{~h}$ a week & 21.1 & 68.2 & 8.8 & 22.6 & 72.0 & 5.4 & \\
\hline \multicolumn{8}{|l|}{ Dieting } \\
\hline Never dieted & 26.6 & 63.4 & 10.0 & 34.6 & 60.7 & 4.7 & \multirow[t]{2}{*}{$\mathrm{M}=<0.001, \mathrm{~F}=<0.001$} \\
\hline Past and present dieters & 7.3 & 65.5 & 27.3 & 12.1 & 75.3 & 12.6 & \\
\hline
\end{tabular}

Note: Cross-tabulations of BMI and other factors; data are expressed as percentages unless otherwise indicated.

* Out of school hours.

behaviour) are shown as adjusted odds ratios (ORs) with 95\% confidence intervals by gender (Table 6) obtained from a parsimonious ordinal regression model. The factors adjusted for, besides those shown in Table 6, included physical activity but excluded cola/soft drinks and time spent watching TV as these were not significant in the bivariate analysis for both boys and girls (Table 5). Girls from East Asia and boys from sub-Saharan Africa (OR 0.4 in each case) were more likely to have a lower BMI than the Western group. Girls seldom consuming chocolate or sweets, full-fat milk and breakfast (OR 2.4, 1.7 and 1.7 , respectively) were more likely to have higher BMI. The likelihood of having higher BMI was greater for both girls and boys who were dieting or had dieted in the past (OR 3.7 and 4.2, respectively). Interaction between dieting and ethnicity was tested using the likelihood ratios and no interaction was observed (data not shown).

\section{Discussion}

Significant ethnic and gender differences in BMI, diet and physical activity were observed. Ethnic differences in socio-economic status were also observed. BMI was not associated with socio-economic factors or physical activity. However, significant associations of BMI were found with ethnicity and dietary habits including dieting.

The main strengths of this study include a populationbased representative sample of ethnic adolescents from Oslo, with wide-ranging health-related questions that enabled us to explore and study associations. The additional access to register data on socio-economic status of parents has been invaluable in studying infrequently reported health inequalities among this group.

The main weakness of the study is inherent in its crosssectional design, where the measures of exposure and effect taken at the same time limit inferences as to causeand-effect relationships ${ }^{18}$, and this is illustrated well in the case of BMI and associated factors. It is difficult ascertain the chronology of events in the association between dieting and BMI. Given that adolescence is a period in the lifecycle of constantly changing body size and shape, longitudinal data would have been extremely valuable. The limitations of self-reported weight and height described by Strauss ${ }^{19}$ and others are well recognised and this bias cannot be ruled out completely. Since BMI does not address degree of fatness, assessment of longterm trends in adiposity needs to be followed up by 
Table 6 Adjusted $^{*}$ odds ratio (OR) and $95 \%$ confidence interval $(\mathrm{Cl})$ for selected factors significantly associated with body mass index of 15- and 16-year-olds from ethnic minorities in Oslo, Norway

\begin{tabular}{|c|c|c|c|c|c|c|}
\hline & \multicolumn{3}{|c|}{ Female $(n=847)$} & \multicolumn{3}{|c|}{ Male $(n=812)$} \\
\hline & OR & $95 \% \mathrm{Cl}$ & $P$-value & OR & $95 \% \mathrm{Cl}$ & $P$-value \\
\hline \multicolumn{7}{|l|}{ Ethnicity } \\
\hline Western countries & 1 & & & 1 & & \\
\hline East Asia/Western Pacific & 0.4 & $0.1-1.0$ & 0.06 & 0.5 & $0.2-1.1$ & 0.12 \\
\hline Indian subcontinent & 0.6 & $0.2-1.2$ & 0.20 & 0.6 & $0.2-1.2$ & 0.19 \\
\hline Sub-Saharan Africa & 0.9 & $0.4-2.2$ & 0.93 & 0.4 & $0.2-1.0$ & 0.05 \\
\hline Middle East/North Africa & 1.2 & $0.6-2.7$ & 0.50 & 0.7 & $0.4-1.7$ & 0.57 \\
\hline Eastern Europe & 1.6 & $0.6-3.8$ & 0.26 & 1.0 & $0.4-2.6$ & 0.86 \\
\hline \multicolumn{7}{|l|}{ Fruit and vegetable index } \\
\hline High & 1 & & & 1 & & \\
\hline Moderate & 1.3 & $0.9-1.8$ & 0.98 & 0.7 & $0.5-1.0$ & 0.08 \\
\hline Low & 0.3 & $0.1-0.6$ & 0.03 & 0.9 & $0.5-1.6$ & 0.70 \\
\hline \multicolumn{7}{|l|}{ Chocolate and sweets consumption } \\
\hline Daily/once or several times a day & 1 & & & 1 & & \\
\hline Weekly/1-6 times a week & 1.4 & $1.1-2.1$ & 0.02 & 1.1 & $0.8-1.6$ & 0.33 \\
\hline Seldom/1-3 times a month & 2.4 & $1.1-5.0$ & 0.01 & 2.1 & $0.9-1.0$ & 0.06 \\
\hline \multicolumn{7}{|l|}{ Full-fat milk } \\
\hline Daily/1-4 glasses a day & 1 & & & 1 & & \\
\hline Weekly/1-6 glasses a week & 1.5 & $1.0-2.3$ & 0.03 & 1.2 & $0.8-1.8$ & 0.25 \\
\hline Seldom/never & 1.7 & $1.1-2.6$ & 0.01 & 1.2 & $0.7-1.9$ & 0.35 \\
\hline \multicolumn{7}{|l|}{ Breakfast consumption } \\
\hline Regular/daily, 5-6 times a week & 1 & & & 1 & & \\
\hline Weekly/1-4 times a week & 1.0 & $0.6-1.4$ & 0.14 & 1.1 & $0.8-1.6$ & 0.43 \\
\hline Seldom/never & 1.7 & $1.1-2.8$ & $<0.001$ & 1.4 & $0.9-2.4$ & 0.13 \\
\hline \multicolumn{7}{|l|}{ Dieting } \\
\hline Never dieted & 1 & & & 1 & & \\
\hline Past and present dieters & 3.7 & $2.6-5.3$ & $<0.001$ & 4.2 & $2.7-6.6$ & $<0.001$ \\
\hline
\end{tabular}

Note: An ordinal regression model was used with body mass index in the same three groups as in Table 5, adjusted for all factors excluding cola/soft drinks and time spent on television/video/computer (not significant from Table 5). Physical activity was not significant in the multivariate analyses and is therefore not shown in this table.

measures such as skinfold thickness, as degree of fatness can differ at the same $\mathrm{BMI}^{20}$.

By categorising groups based on ethnicity rather than race we were able to include the social and cultural aspects ignored by racial classifications. These broad categories of ethnicity still limit interpretations, but sample size constrained further divisions. Another drawback is that the food-frequency questionnaire has not been validated for ethnic minorities, but most of the questions have been validated for Norwegians. As we do not have adequate data on parents' length of stay in Norway or assimilation into Norwegian society, it is rather difficult to explain the adolescents' food choices and rationale for related behaviour in this context.

Our study documents ethnic differences in lifestyle factors among adolescents that warrant special attention, particularly in the evolving multi-ethnic societies of Europe. These differences with regard to minorities that are at a crossroads must be considered when strategies for health education, promotion and prevention are being developed, because these might be both crucial and successful in delaying or preventing the onset of chronic disease in adult life. Ethnic differences in cardiovascular risk factors have already been documented ${ }^{21}$. BMI in adults is frequently associated with socio-economic factors $^{22}$, but despite socio-economic inequalities among the ethnic adolescents, this association was not manifested herein. These findings concur with those of Winkleby et al., where ethnic differences remained significant after accounting for socio-economic status and age ${ }^{21}$. Several studies show that immigrants from the Indian subcontinent are likely to be overweight in adult life ${ }^{12}$, and in light of this and the burgeoning diabetes epidemic, our study illustrates the need for longitudinal studies to follow the course of the change in BMI from adolescence.

BMI as the ideal measure of obesity in adolescents is refutable but the International Obesity Task Force ${ }^{23,24}$ concluded that it is a reasonable measure of fatness. Different classifications and definitions of overweight and obesity are currently in use 25,26 . However, in order to make comparisons with other Norwegian studies, the work of Engeland et $a l^{3}$ and other multi-ethnic studies (USA primarily), we have used the age- and sex-specific growth charts from CDC/NCHS to categorise adolescents into BMI groups.

Gender differences in BMI are known and wellestablished for adults and adolescents, and our findings were no different from other studies ${ }^{27}$. Strauss and others discuss gender biases in reporting of weight and height as being relatively small and concluded that their results were fairly reliable for predicting obesity-related morbidities and behaviours ${ }^{19}$. Klasson-Heggebo et al. recorded heights and weights from the same sampling universe as our study and marginal deviations were observed ${ }^{28}$. Boys 
weighed $62.4 \pm 9.6 \mathrm{~kg}$ in their study, compared with $61.9 \pm 10.4 \mathrm{~kg}$ in ours. While we lay no claims to this study as a validation of our work, it suffices to indicate that, despite apparent limitations of self-reported weights and heights, individuals are less likely to be misclassified in the BMI groups.

Studying other factors influencing growth and development in the adolescent years - such as foetal undernutrition $^{29}$, birth weight, childhood undernutrition and disease, to name a few - would have enabled us to study and analyse the evolution of BMI better, but is presently beyond the scope of this study.

While most other studies ${ }^{30,31}$ have focused to a large extent on racial differences (black, white, Hispanic) of BMI in adolescence, ethnic differences have scarcely been studied. Given that adolescents are at a developmental stage where cultural factors play an important role, ethnicity particularly in the case of immigrants should be given importance. Norway's previously homogeneous population has over the past 30 years evolved into a multicultural society that warrants the examination of ethnic health inequalities.

An ethnic dichotomy of overweight and underweight is evident, with the Western, Eastern European and Middle East/North Africa groups having a greater likelihood of being overweight whereas those from the Indian subcontinent, sub-Saharan Africa and East Asia tend towards underweight. However, the overall proportion of overweight was not high as might be expected, especially in comparison with adolescents in the USA and other European countries.

Others have studied and demonstrated a high prevalence of overweight and obesity among adult immigrants from the Indian subcontinent in the $\mathrm{UK}^{32}$. Interestingly, our study shows that the proportion of overweight adolescents from the Indian subcontinent, compared with adults (first-generation immigrants), is very low (Kumar, forthcoming). Further comparisons with studies from the Indian subcontinent show that results are not very different from urban areas in terms of overweight, but lower in terms of underweight especially in rural areas ${ }^{33}$.

Ethnic differences in health outcomes have received growing attention over the past two decades. Bhopal ${ }^{14}$ discusses ethnicity and race as the most controversial and difficult of all epidemiological variables, yet integral to modern epidemiology and public health. Ethnic groups are maintained through culture and socialisation ${ }^{34}$, but are hard to classify and define as this presumes social homogeneity. Assigning ethnic groups by common food cultures overcomes some of the shortcomings of determining ethnicity geographically, i.e. by country of birth and by race.

Social inequalities in adolescent health are scarcely elaborated in the literature ${ }^{35}$. These differences are apparent in our sample especially between the Western and non-Western groups, where the majority from nonWestern countries belonged to the lower classes. Statistics Norway's education register based on self-reported data for those educated outside Norway raises concerns about reliability. Information on parents' education and income, obtained from such registers, could therefore be inaccurate predictors of socio-economic position. Notwithstanding these limitations, facts suffice to point towards an unusually high proportion of uneducated mothers among the ethnic minorities. The majority of immigrants live in the eastern, more disadvantaged part of the city of Oslo, known and described as the East-West divide $^{17}$. This concurs with the contention that ethnic minority people are concentrated in particular geographic locations that are likely to be more deprived areas ${ }^{34}$. Interestingly, our analysis reveals that most immigrants regardless of social class live in the eastern part of this city and this could be attributed to them being more comfortable among their own.

Besides socio-economic differences, lifestyle factors also varied with ethnicity. Consistent with their food traditions, the Mediterranean group consumed more fruits and vegetables. On the other hand, the Indian subcontinent group's low consumption of fruits and vegetables deviates from the traditional diet abundant in vegetables and fruits, suggesting post-migration changes. Our recent in-depth study among Pakistani women in Oslo confirmed dietary changes that could be attributable to their length of stay in Norway (unpublished). Ethnic adolescents are in double jeopardy by persisting with unhealthy habits from their minority culture and acquiring unhealthy habits from the majority. This is illustrated well in the case of boys from the Indian subcontinent, with a high consumption of both full-fat milk and cola/soft drinks.

The association of skipping breakfast with higher BMI may be explained by compensation with energy-rich foods later, snacking or dieting. The inverse relationship with chocolate/sweets and full-fat milk consumption and BMI, observed in other studies as well ${ }^{36}$, indicates complex underlying behavioural processes. Underreporting among overweight individuals, well-documented in other studies ${ }^{37}$, is one likely explanation. If reporting is assumed to be correct, then overweight adolescents could be avoiding these foods to achieve weight reduction and this could occur even among those who do not think of themselves as dieters. Vander Wal and Thalen ${ }^{38}$ observed that obese children, especially girls, were more likely to engage in dieting behaviours, to express concern about their weight, to restrain their eating and to exhibit more dissatisfaction with their body image than average-weight children. We concur with these authors, as we found that the likelihood of dieting increased among those who are overweight. However, owing to the limitations of a crosssectional dataset, it is impossible to state categorically that weight gain is the reason for dieting, because dieting is also well-established among those with normal weight 
and underweight. Moreover, dieting is a widespread teenage phenomenon that could be independent of weight gain, particularly in the case of girls.

Gordon-Larsen and co-workers have documented ethnic differences in physical inactivity? 9 . Unlike the association between low levels of physical activity and high BMI in other studies ${ }^{36,39}$, surprisingly no association was found in our study (regression analysis) despite high levels of inactivity and sedentary activities. This might indicate that either the variation in physical inactivity was minimal and therefore insignificant or that the measurement of physical activity was inaccurate and inappropriate. Pratt et al. reported that estimates of physical activity are less reliable and valid for adolescents than for adults ${ }^{40}$.

Despite ethnic variations, food and drink habits associated with adolescent behaviour such as snacking, skipping meals and dieting are similar to those found in other studies in Europe ${ }^{4}$, and in this regard ethnic minorities are very much like their European peers. Unhealthy behaviour, particularly those that impact on the development of food habits, and physical activity in adolescence need to be further examined. Studying the underlying processes involved in making these choices, and qualitative studies among adolescents to gain a better understanding, should form an essential part of future research.

In conclusion, ethnic differences in BMI in adolescence need careful consideration in light of consequences such as nutrition-related chronic diseases in adulthood. It is therefore essential that second-generation immigrants, in particular those from the Indian subcontinent, known to be at exceptionally high risk for developing chronic diseases in adulthood such as the metabolic syndrome, be followed over time to determine and identify risk factors and develop preventive strategies. Lastly, the Oslo Health Study has provided a unique opportunity to study ethnic minorities and elucidate differences in diet, physical activity and nutrition, thereby reaffirming that the ethnicity variable is crucially important in epidemiological studies and important science ${ }^{14}$ throughout the lifecycle including adolescence.

\section{Acknowledgements}

The authors would like to thank Magne Thoresen for statistical advice, Anders Engeland and Anne Johanne Søgaard for valuable comments, Annhild Mosdøl for questionnaire development and Ånen Ringard and Zumin Shi for assistance.

\section{Contributors}

The data collection was conducted as part of the Oslo Health Study 2000-2001 in collaboration with the National Health Screening Service of Norway, now the Norwegian Institute of Public Health. B.N.K., G.H.-O. and M.W. contributed to the conception and design of the study.
B.N.K. and N.L. analysed the data, and all authors contributed to the interpretation of the data. B.N.K. drafted the paper and G.H.-O., M.W. and N.L. commented upon and revised drafts. All authors gave their approval to the final version submitted for publication.

\section{Funding support}

The Oslo Health Study was funded by the Norwegian Institute of Public Health, the University of Oslo and the Municipality of Oslo. B.N.K.'s doctoral grant was funded through the Norwegian Research Council, and the Norwegian Directorate for Health and Social Affairs, Nutrition Division financially supported this work.

\section{Competing interests}

None declared.

\section{References}

1 United Nations Administrative Committee on Coordination, Sub-committee on Nutrition (ACC/SCN). Fourth Report on the World Nutrition Situation. Geneva: ACC/SCN in collaboration with International Food Policy Research Institute, 2000.

2 Lien N, Lytle LA, Klepp KI. Stability in consumption of fruit, vegetables, and sugary foods in a cohort from age 14 to age 21. Preventive Medicine 2001; 33(3): 217-26.

3 Engeland A, Bjørge T, Selmer RM, Tverdahl A. Height and body mass index in relation to total mortality. Epidemiology 2003; 14(3): 293-9.

4 Samuelson G. Dietary habits and nutritional status in adolescents over Europe. An overview of current studies in the Nordic countries. European Journal of Clinical Nutrition 2000; 54(Suppl. 1): S21-8.

5 Rolland-Cachera MF, Bellisle F, Deheeger M. Nutritional status and food intake in adolescents living in Western Europe. European Journal of Clinical Nutrition 2000; 54(Suppl. 1): S41-6.

6 Lowry R, Wechsler H, Galuska DA, Fulton JE, Kann L. Television viewing and its associations with overweight, sedentary lifestyle, and insufficient consumption of fruits and vegetables among US high school students: differences by race, ethnicity, and gender. Journal of School Health 2002; 72(10): 413-21.

7 Steinbeck KS. The importance of physical activity in the prevention of overweight and obesity in childhood: a review and an opinion. Obesity Reviews 2001; 2(2): 117-30.

8 Kaur H, Choi WS, Mayo MS, Harris KJ. Duration of television watching is associated with increased body mass index. Journal of Pediatrics 2003; 143(4): 506-11.

9 Gordon-Larsen P, McMurray RG, Popkin BM. Adolescent physical activity and inactivity vary by ethnicity: The National Longitudinal Study of Adolescent Health. Journal of Pediatrics 1999; 135(3): 301-6.

10 Andersen LF, Nes M, Sandstad B, Bjorneboe GE, Drevon CA. Dietary intake among Norwegian adolescents. European Journal of Clinical Nutrition 1995; 49(8): 555-64.

11 World Health Organization (WHO). Health Behaviour among Young People. Health Policy for Children and Adolescents Series, Report No. 1. Geneva: WHO, 2000.

12 Bhopal R, Unwin N, White M, Yallop J, Walker L, Alberti KG, et al. Heterogeneity of coronary heart disease risk factors in Indian, Pakistani, Bangladeshi, and European origin populations: cross sectional study. British Medical Journal 1999; 319(7204): 215-20. 
13 Kimm SY, Barton BA, Obarzanek E, McMahon RP, Sabry ZI, Waclawiw MA, et al. Racial divergence in adiposity during adolescence: The NHLBI Growth and Health Study. Pediatrics 2001; 107(3): E34.

14 Bhopal R. Is research into ethnicity and health racist, unsound, or important science? British Medical Journal 1997; 314(7096): 1751-6.

15 Lie B. Innvandring og innvandrere 2002. Oslo: Statistisk sentralbyrå, 2002.

16 Kuczmarski RJ, Ogden CL, Grummer-Strawn LM, Flegal KM, Guo SS, Wei R, et al. CDC growth charts: United States. Advance Data 2000; (314): 1-27.

17 Rognerud MA, Kruger O, Gjertsen F, Thelle DS. Strong regional links between socio-economic background factors and disability and mortality in Oslo, Norway. European Journal of Epidemiology 1998; 14(5): 457-63.

18 Bhopal RS. Concepts of Epidemiology: An Integrated Introduction to the Ideas, Theories, Principles, and Methods of Epidemiology. Oxford: Oxford University Press, 2002.

19 Strauss RS. Comparison of measured and self-reported weight and height in a cross-sectional sample of young adolescents. International Journal of Obesity and Related Metabolic Disorders 1999; 23(8): 904-8.

20 Jebb SA, Lambert J. Overweight and obesity in European children and adolescents. European Journal of Pediatrics 2000; 159(Suppl. 1) S2-4.

21 Winkleby MA, Robinson TN, Sundquist J, Kraemer HC. Ethnic variation in cardiovascular disease risk factors among children and young adults: findings from the Third National Health and Nutrition Examination Survey, 1988-1994. Journal of the American Medical Association 1999; 281(11): 1006-13.

22 Sturm R, Wells KB. Does obesity contribute as much to morbidity as poverty or smoking? Public Health 2001; 115(3): 229-35.

23 World Health Organzation (WHO). Obesity: Preventing and Managing the Global Epidemic. Report of a WHO Consultation on Obesity, Geneva, 3-5 June 1997. Geneva: WHO, 1998; 1-276.

24 Bellizzi MC; Dietz WH. Workshop on childhood obesity: summary of the discussion. American Journal of Clinical Nutrition 1999; 70(1): 173S-5S.

25 Must A, Jacques PF, Dallal GE, Bajema CJ, Dietz WH. Longterm morbidity and mortality of overweight adolescents. A follow-up of the Harvard Growth Study of 1922 to 1935. New England Journal of Medicine 1992; 327(19): 1350-5.

26 Cole TJ, Bellizzi MC, Flegal KM, Dietz WH. Establishing a standard definition for child overweight and obesity worldwide: international survey. British Medical Journal 2000; 320(7244): 1240-3.

27 Guo SS, Wu W, Chumlea WC, Roche AF. Predicting overweight and obesity in adulthood from body mass index values in childhood and adolescence. American Journal of Clinical Nutrition 2002; 76(3): 653-8.

28 Klasson-Heggebo L, Andersen K. Gender and age differences in relation to the recommendations of physical activity among Norwegian children and youth. Scandinavian Journal of Medicine \& Science in Sports 2003; 13(5): 293-8.

29 Eriksson JG, Forsen T, Tuomilehto J, Winter PD, Osmond C, Barker DJ. Catch-up growth in childhood and death from coronary heart disease: longitudinal study. British Medical Journal 1999; 318(7181): 427-31.

30 Lytle LA, Himes JH, Feldman H, Zive M, Dwyer J, Hoelscher $\mathrm{D}$, et al. Nutrient intake over time in a multi-ethnic sample of youth. Public Health Nutrition 2002; 5(2): 319-28.

31 Berkey CS, Rockett HR, Field AE, Gillman MW, Frazier AL, Camargo CA Jr, et al. Activity, dietary intake, and weight changes in a longitudinal study of preadolescent and adolescent boys and girls. Pediatrics 2000; 105(4): E56.

32 Shetty PS, McPherson K, eds. Diet, Nutrition and Chronic Disease: Lessons from Contrasting Worlds. New York: John Wiley \& Sons, 1997; 59-75.

33 Venkaiah K, Damayanti K, Nayak MU, Vijayaraghavan K. Diet and nutritional status of rural adolescents in India. European Journal of Clinical Nutrition 2002; 56(11): 1119-25.

34 Macbeth H, Shetty PS, eds. Health and Ethnicity. London: Taylor \& Francis, 2001; 41-58.

35 Saxena S, Majeed A, Jones M. Socioeconomic differences in childhood consultation rates in general practice in England and Wales: prospective cohort study. British Medical Journal 1999; 318(7184): 642-6.

36 De Vito E, La Torre G, Langiano E, Berardi D, Ricciardi G. Overweight and obesity among secondary school children in central Italy. European Journal of Epidemiology 1999; 15(7): 649-54.

37 Garaulet M, Martinez A, Victoria F, Perez-Llamas F, Ortega RM, Zamora S. Difference in dietary intake and activity level between normal-weight and overweight or obese adolescents. Journal of Pediatric Gastroenterology and Nutrition 2000; 30(3): 253-8.

38 Vander Wal JS, Thelen MH. Eating and body image concerns among obese and average-weight children. Addictive Behaviors 2000; 25(5): 775-8.

39 Andersen RE, Crespo CJ, Bartlett SJ, Cheskin LJ, Pratt M. Relationship of physical activity and television watching with body weight and level of fatness among children: results from the Third National Health and Nutrition Examination Survey. Journal of the American Medical Association 1998; 279(12): 938-42.

40 Pratt M, Macera CA, Blanton C. Levels of physical activity and inactivity in children and adults in the United States: current evidence and research issues. Medicine and Science in Sports and Exercise 1999; 31(Suppl. 11): S526-33. 